\section{ORGANIC CHEMISTRY}

\section{Carbon dioxide conversions}

The abundance of carbon dioxide makes it an attractive starting material for petrochemical-free synthesis of organic chemicals. But turning carbon dioxide into useful molecules has proved difficult. Thibault Cantat and his colleagues at the French Alternative Energies and Atomic Energy Commission in Gif-sur-Yvette have devised a way to generate a potentially large number of chemicals directly from $\mathrm{CO}_{2}$ without the need for high pressure, organic solvents or expensive and toxic metal catalysts.

Using an organic catalyst, the team managed, in one step, to reduce $\mathrm{CO}_{2}$ by replacing the oxygen with hydrogen, and also to attach another atom to the carbon. Previous techniques could only accomplish one or other of these tasks. The authors showed that they could synthesize high yields of a variety of formamides using amines, $\mathrm{CO}_{2}$, silanes as reducing agents and the organic catalyst.

Chemicals other than amines may be obtained by tuning the reagents, the authors suggest.

Angew. Chem. http://dx.doi. org/10.1002/anie.201105516 (2011)

\section{NEURODEVELOPMENT}

\section{Cell source for brain disorder}

Children with a genetic disease called tuberous sclerosis complex (TSC) develop benign growths in various tissues, including the central nervous system where the growths cause epilepsy, autism and mental retardation.

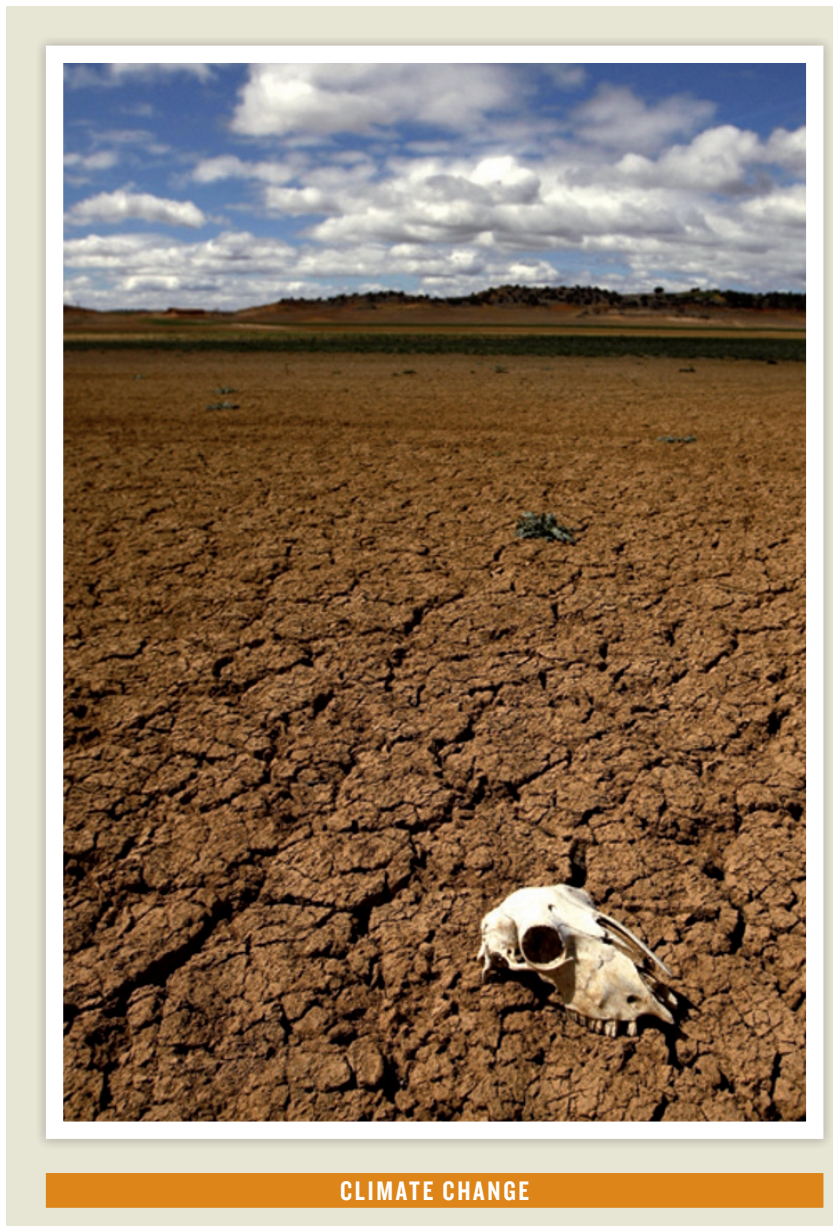

\title{
Mediterranean drying
}

Global warming is contributing to a rise in both the magnitude and the frequency of wintertime droughts in the Mediterranean region. Of the 12 driest winters it has experienced since 1902, 10 occurred during the past 20 years.

Martin Hoerling and his colleagues at the US National Oceanic and Atmospheric Administration in Boulder, Colorado, used precipitation and ocean surface temperature data, along with climate models, to investigate drought conditions dating back to 1902 . The team found that climate change can explain roughly half the trend towards drier conditions during this period.

In particular, the team linked wintertime droughts to rising sea surface temperatures in the tropical oceans.

J. Clim. http://dx.doi.org/10.1175/JCLI-D-11-00296.1 (2011)

Rossella Galli at the San

Raffaele Scientific Institute in

Milan, Italy, and her colleagues have pinpointed a population of embryonic cells in mice that, when mutated, bring about signs of the disease.

The researchers engineered mice in which they could inactivate the gene Tsc1, which is linked to TSC, in a group of embryonic neural stem cells.
These cells give rise to the brain regions affected by the disorder. Shortly after birth, the mice had lesions in those brain areas and suffered from seizures.

The team then isolated these mutant stem cells and administered rapamycin, a molecule that blocks a pathway, called mTOR, that is overactive in TSC. This reduced the cells' size and other defects. The authors conclude that mTOR activation must be finely tuned in these cells for normal neural development.

Cell Stem Cell 9, 447-462 (2011)

PALAEONTOLOGY

\section{Ancient creature's surprising sight}

The fossilized compound eyes of a crustacean that lived around half a billion years ago reveal a surprisingly sophisticated visual system that was probably able to detect motion and gauge distance in many directions.

Resembling a tiny mace, the eye consists of four distinct areas atop a stalk measuring at most 0.5 millimetres in length. The four overlapping zones scanned mainly forwards, backwards, to the outer sides and inner sides, respectively.

Brigitte Schoenemann at the University of Bonn in Germany and her colleagues say that the eyes of Henningsmoenicaris scutula lacked fine resolution owing to a relatively low number of visual units. However, the eyes probably allowed the creature to detect moving objects such as prey - by tracking them as they crossed from one visual sector to another - while also giving the crustacean a wraparound view of the world.

Proc. R. Soc. B http://dx.doi. org/10.1098/rspb.2011.1888 (2011) 